\title{
Qualifying the Sunpower M87N Cryocooler for operation in the AMS-02 Magnetic Field
}

\author{
Shuvo Mustafi, Stuart Banks, Kim Shirey, Susan Breon \\ NASA Goddard Space Flight Center \\ Greenbelt, MD, USA 20771
}

\begin{abstract}
The Alpha Magnetic Spectrometer-02 (AMS-02) experiment uses a superfluid helium dewar to cool a large superconducting magnet. The outer vapor-cooled shields of the dewar are to be held at $80 \mathrm{~K}$ by four Sunpower M87N cryocoolers. These cryocoolers have magnetic components that might interact with the external applied field generated by the superconducting magnet, thereby degrading the cryocoolers' performance. Engineering models of the Sunpower M87 have been qualified for operation in a magnetic environment similar to the AMS-02 magnetic environment.
\end{abstract}

Although there was no noticeable performance degradation at field levels that were comparable to AMS-02 field levels, there appears to be a small performance degradation at higher field levels. It was theorized that there were three possible issues related to these performance losses at high magnetic fields: i) induced piston rubbing on the cylinder wall due to forces and torques on the linear motor due to the applied magnetic fields; ii) Magnetic hysteretic and/or eddy current damping of the balancer due to its motion in the applied magnetic fields; iii) Inductance losses in motor due to the applied magnetic field.

The experiments conducted at the Massachusetts Institute of Technology (MIT) cyclotron facility in June 2002 were designed to test these. Tests were performed over a range of field levels that were lower, comparable, and higher than the field levels that the cryocoolers will experience in the AMS- 02 operating environment. This paper describes the experiments and the inferences derived from them.

\section{Introduction}

The AMS - 02 mission is an International Space Station (ISS) based experiment to detect dark matter and anti-matter. Large superconducting magnets cooled by superfluid helium, contained in a dewar, will be used to direct the cosmic particles through a series of detectors that will measure the momentum and charge of the particles. The outer vapor cooled shield of the dewar will be cooled using four Sunpower M87N cryocoolers. The cryocoolers will decrease the rate of evaporation of the superfluid helium from the dewar and thereby extend the mission duration. [1] 
The Sunpower M87N is a Stirling cycle linear compressor cooler. The linear compressor has a piston that is driven by a linear motor consisting of a fixed solenoid and a moving magnet-ring. The magnet-ring has a cylinder with a set of magnetic tablets arranged symmetrically around the surface of the cylinder such that the dipoles are oriented in a radial direction.

\section{Performance Degradation}

We were concerned that interactions between the AMS-02 magnetic field and the cryocooler motor might affect the cooling performance of the coolers. As we reported previously the cryocoolers experienced about a $1-4 \%$ rise in temperature at fields $1.5-2$ times than the predicted AMS-02 field levels. [2] This paper presents the results of further investigation into the loss of performance.

It was theorized that there were three issues related to these performance losses at high magnetic fields:

1. Induced piston rubbing on the cylinder wall due to forces and torques on the linear motor due to the applied magnetic fields.

2. Magnetic hysteretic and/or eddy current damping of the balancer due to its motion in the applied magnetic fields.

3. Inductance losses in the motor due to the applied magnetic field.

The experiments conducted in June 2002 were designed to test these theories in the MIT cyclotron facility at field levels that were comparable to the perpendicular field levels in the AMS-02 operating environment. 


\section{Co-ordinate transformation of the AMS-02 field to a cryocooler based system}

Each of the AMS-02 cryocoolers will experience different field strengths and field gradients depending on its location relative to the superconducting magnets. The superconducting magnets are being designed and built by Space Cryomagnetics, Ltd. (SCL), in Culham, England. Using their model of the AMS-02 magnet, SCL generated values of the magnetic field along the three axes at 50 grid points specified for each cryocooler (see Figure 1).[2] Before embarking on testing at MIT, the magnetic field values given in AMS-02 coordinates were translated to values in a cryocooler-based coordinate system. These values, along with calculated values for the field gradients, were used to generate magnetic fields at MIT that are similar to the magnetic field that the cryocoolers would experience on AMS-02 (see Table 1).

\section{Behavior of the magnet-ring assembly in an external magnetic field}

It was essential to investigate how the cryocooler magnet-ring would respond when operated within a large external magnetic field. Tests at the Goddard Space Flight Center indicated that the forces and torques on the magnet-ring depended not only on the magnitude of the magnetic field but also the gradient of the magnetic field. Tests also indicated that although the magnetic segments on the magnet-ring are arranged symmetrically around the magnet-ring cylinder, they are in fact not magnetized to the same level due to manufacturing variability - hence the magnet-ring assembly is not magnetized symmetrically. This means that the magnet-ring assembly will tend to rotate to its preferred orientation when introduced into the AMS-02 magnetic field.

\section{Testing at the MIT Cycolotron Facility}

The tests that were carried out at this facility were as follows: 
A. The magnetic field around the experiment environment was mapped prior to placing any test articles in the field.

B. The forces and torques on a stationary Sunpower M87N magnet-ring assembly were measured to investigate the possibility that the piston and cylinder might be rubbing.

C. Other potential reasons for performance degradation were investigated

1. The magnetic hysteretic and/or eddy current damping effects on the Sunpower M87N passive balancer.

2. The inductance losses in the Sunpower M87N motor.

\section{Mapping and comparing test field to AMS-02 field}

Table 1 compares the magnetic field properties (the magnitude and gradients) expected on the AMS-02 experiment to field properties the M87N cryocooler was exposed to during testing at MIT. It is impossible to replicate the AMS-02 field completely without replicating the AMS-02 magnet; therefore the focus of was on testing at field levels that would most likely affect the performance of the cryocooler. The most influential magnetic field property in this case is the field that is perpendicular to the axis of the cryocooler because the perpendicular field is the strongest field component experienced by the cryocooler in the AMS-02 environment. The engineering models (EM) of the Sunpower M87 cryocoolers were tested at field levels that were at $0,0.5,1$, 1.5 and 2 times the expected AMS-02 perpendicular field levels. Table 1 shows some of the field properties that the EM cryocooler was exposed to (column 3) in comparison to the expected field properties in the presence of the AMS-02 superconducting magnet (column 2). Column 4 shows the location where these field properties were measured as 
defined by the Motor Node Set in Figure $1(-\mathrm{y}$ is located left of center in the set of nodes). Column 5 shows a comparison of whether the values obtained during testing at MIT were over or under the predicted AMS-02 field levels. Testing was done at up to 2 times $(0.090 \mathrm{~T})$ the predicted perpendicular AMS-02 magnetic fields, and up to 2.2 times $(0.065 \mathrm{~T})$ the predicted axial AMS-02 magnetic field levels without significant reduction in cryocooler performance. Although the field properties at MIT were not an exact replication of the field levels for AMS-02, almost all the field properties that are most influential to the behavior of the cryocooler were exceeded in the MIT experiment.

\section{Forces and torques induced by an external applied field on the M87N motor}

Motivation

Lateral forces and torques are generated on the $\mathrm{M} 87 \mathrm{~N}$ motor by the applied magnetic field. The gas-bearings on the M87N cryocooler will need to counteract these forces and torques. If the bearings are unable to do so the piston might start rubbing against the cylinder wall. This may cause the cryocooler to fail over time. The force $(\vec{F})$ on the magnet-ring is given by:

$$
\vec{F}=\vec{M} \bullet \nabla \vec{B}_{\text {applied }}
$$

where $\vec{M}$ is the magnetic dipole moment of the magnet-ring in an external magnetic

field. $\quad \vec{B}_{\text {applied }}$ is the external applied field.[3]

The torque ( $\vec{T}$ ) on the magnet-ring can be calculated from:

$$
\vec{T}=\vec{M} \times \vec{B}_{\text {applied }}
$$

The above equations ignore quadropole and higher order effects.

The magnetic dipole moment $(\vec{M})$ is given by:

$$
\vec{M}=\vec{M}_{0}+\Delta \vec{M}
$$




$$
\Delta \vec{M}=f\left(\vec{B}_{\text {applied }}, \nabla \vec{B}_{\text {applied }}\right)
$$

Equations (3)-(4) describe the dependence of the magnetic dipole moment on both the applied field and the gradient of the applied field. $\quad \vec{M}_{0}$ is the dipole moment that ferromagnetic material displays in the absence of a field. $\Delta \bar{M}$, the additional dipole moment induced in the presence of a magnetic field, $\Delta \vec{M}$ is a function of the applied field and the gradient of the applied. The force and torque on a ferromagnetic body are thus a function of both the applied field and the applied field gradient as was qualitatively observed during the preparation for the tests at MIT.

A test was performed to measure the forces and torques that are induced on the cryocooler magnet-ring assembly. The data acquired from this test would give an understanding of the magnitudes of the forces and torques induced on the motor by an external magnetic field.

\section{Method}

Figure 2 shows the assembly used to measure the forces on the M87N magnetring. The magnet-ring assembly is held in place using non-magnetic arms and a central non-magnetic support rod. The arms are attached to calibrated force-transducers at the other end (at the frame). The transducers are not affected by the external magnetic field and this was independently verified by calibrating the transducers with weights in an external magnetic field. The transducers can measure forces in both compression and tension. This set-up enables the transducers to measure forces in all three co-ordinate axes. The whole assembly was placed in the cyclotron magnetic field, and the voltages recorded from each transducer enabled the calculation of the force distribution on the magnet-ring assembly after the gravity effects were zeroed out. With this data it was 
possible to calculate the net magnetic force, the point of application of this force, and the resulting torque experienced by the cryocooler magnet-ring assembly for different field strengths as well as different orientations of the cryocooler magnet-ring assembly relative to its preferred orientation in the magnetic field.

Results

Figure 3 shows the magnitude of the net magnetic force imparted on the M87N magnet-ring assembly for varying applied fields at the ring's preferred orientation, as well as three angles of rotation about its axis. The orientation of the magnet-ring has a substantial effect on the net force experienced by the magnet-ring. The force imparted for all orientations increases with increasing magnetic field.

Figure 4 shows the variation in the magnitude of the net magnetic torque on the magnet-ring with the applied field for the preferred orientation. The torques experienced by the magnet-ring also seem to increase with increasing field. There is a also a dependence of the magnitude of the net torque on the orientation of the magnetring as well, although the dependence does not seem to be as well defined. Figure 5 shows the variation in the magnitude of the net magnetic torque on the M87N magnetring assembly for various orientations. The general trend for these is that the magnetic torque increases with the field, although there appears to be anomalies to this trend, as can be seen for the 120 degree orientation data in Figure 5.

\section{Explanation of Results}

The net force on the magnet-ring assembly increases with increasing field as can be predicted by equation (1). The net force also varies with the orientation of the magnet-ring. In general the net torque on the assembly increases with the increasing field as predicted by equation (2), although anomalies to this trend exist for some of the 
orientations of the magnet-ring. The anomalies in the cases where the magnet-ring was not in the preferred orientation can perhaps be attributed to additional torques the magnet-ring assembly experienced that were trying to rotate the magnet-ring assembly to the preferred orientation. Although these anomalies and the dependence of the force and torque on the orientation were curious effects we did not further investigate their occurrence due to time constraints.

In this setup the orientations were restricted but in the AMS-02 setup, the orientation won't be restricted. No matter what orientation the magnet-ring is outside the AMS-02 magnetic field, when it is inside the magnetic field, it will reorient itself to its preferred orientation and experience force and torque levels that are of the order of the force and torques experienced in the preferred orientation direction during this experiment. In the preferred orientation there is a definite increase in the force and torques in an increasing magnetic field.

The reaction forces on the bearings resulting from the external magnetic field were comparable to the static gravitational loads. Other forces in the operational cryocoolers are larger than gravitational loads. Hence the magnetic forces and torques imparted on the magnet-ring by an external magnetic field should be well compensated for by the gas bearings.

Unfortunately there is no easy way to do this test on an operating motor. Forces on the stationary cryocooler magnet are an approximation to the forces that an operational cryocooler magnet would experience hence this is a good initial test 


\section{Eddy Current and/or Magnetic Hysteretic Damping of the M87N balancer}

During the tests conducted in March 2002 it was noticed that the presence of the balancer affected the performance of the cryocooler. An experiment was conducted to determine if eddy current and/or magnetic hysteresis were responsible for damping of the M87N balancer, thereby causing some performance degradation on the cryocooler. The cryocooler was tested in the external MIT magnetic field with balancers made of different materials to distinguish between these two effects. Figure 6 shows the M87N cold tip temperature at increasing applied magnetic field levels for the different balancer materials. The temperature rise with increasing magnetic fields is greater for the magnetic steel (prone to magnetic hysteretic damping) balancer than it is for copper (prone to eddy current damping) balancer. From the tests we conclude that most of the balancer losses are due to magnetic hysteretic damping and not eddy current damping.

\section{Inductance losses in M87N motor due to applied magnetic field}

The M87N motor solenoid and laminates form an inductor. The inductance drops when the applied magnetic field is high enough to saturate the laminates. This reduction in inductance affects the cryocooler stroke, which then results in lowered cryocooler performance.

An experiment was conducted to see if the inductance of the coil does in fact diminish with increasing applied field. Figure 7 shows the measured dependence of the M87N motor inductance on the applied magnetic field. The inductance drops with increasing field levels, but the effect is small Shielding the cryocooler motor coils from the applied magnetic field could reduce these losses, but the increased weight required for the shielding is not justified by the performance gained from shielding the cryocooler. 


\section{Conclusions}

All indications from testing are that there is no rubbing between the piston and the cylinder wall when the M87N is operated at levels up to twice the expected perpendicular field levels in the AMS-02 scenario (and up to 2.2 times the expected axial field levels). The magnetic forces and torques on the M87N magnet-ring assembly seem to be at levels that can be tolerated by the gas-bearings at even twice the expected field levels, at least in the non-operational case. The balancer tests gave overwhelming evidence that most of the balancer damping that was causing the performance degradation was a result of magnetic hysteretic damping as opposed to eddy current damping. We have switched to a non-magnetic steel balancer to solve this problem. The inductance test conclusively proved that the applied magnetic field reduced the inductance from the motor, and hence degraded the cryocooler performance. Although this problem could be resolved with shielding, it was determined that the performance improvement that would be seen with shielding would not justify the additional weight required for the shielding.

There is very little noticeable performance degradation at field levels that are similar to what will be experienced under the influence of the AMS-02 superconducting magnets. In light of the above experiments, the performance degradation at higher field levels has been studied and is now fairly well understood. We are thus convinced that the M87N cryocooler will not have any major performance problems operating in the AMS-02 superconducting magnet environment. Testing the cryocooler operation in the presence of the actual AMS-02 magnet is the only way to determine with absolute certainty that there will be little or no performance degradation in orbit. The AMS-02 
superconducting magnets are being made in England. The Sunpower M87N will be tested in the presence of the actual AMS-02 magnetic field in 2004/2005.

These tests to study the performance degradation of the Sunpower M87N cryocooler have provided valuable insight into the operation of a linear motor type cryocooler in high magnetic fields. Future space flight missions such as the VAriable Specific Impulse Magnetoplasma Rocket (VASIMR) that will also be using cryocoolers in the presence of strong superconducting magnets will be able to use the knowledge and experience gained from this endeavor for their space-flight qualification process.

\section{Acknowledgments}

The authors wish to thank Rob Boyle, Brent Warner, and Henning Leidecker of NASA -

Goddard Space Flight Center for their guidance during this investigation.

\section{References}

1. Shirey, K.A et al., "Space Flight Qualification Program for the AMS-02 Commercial Cryocoolers," Cryocoolers 12, Kluwer Academic/Plenum Publishers, New York (2003), pp. 37-44

2. Breon, S.R., et al., "Operation of a Sunpower M87N Cryocooler in a Magnetic Field," Cryocoolers 12, Kluwer Academic/Plenum Publishers, New York (2003), pp. 761-769 3.Griffiths, D.J., Introduction to Electrodynamics $3^{\text {rd }}$ ed., 1999, Prentice Hall.

4. Bertotti, Giorgio, Hysteresis in Magnetism, 1998, Academic Press 
Tables

\begin{tabular}{|c|c|c|c|c|}
\hline Field Property & $\begin{array}{l}\text { Predicted Maximum } \\
\text { AMS- } 02 \text { Field } \\
\text { Property }\end{array}$ & $\begin{array}{c}\text { Field the M87N was at least } \\
\text { tested to at MIT }\end{array}$ & Location & Comparison \\
\hline$B x$ & $0.0296 \mathrm{~T}$ & $0.0650 \mathrm{~T}$ & Motor:-y & over \\
\hline By & $0.0374 \mathrm{~T}$ & $0.0010 \mathrm{~T}$ & Motor:-y & under \\
\hline $\mathrm{Bz}$ & $0.0399 \mathrm{~T}$ & $0.0900 \mathrm{~T}$ & Motor:-y & over \\
\hline Byz & $0.0547 T$ & $0.0900 \mathrm{~T}$ & \multicolumn{2}{|l|}{ Motor:-y } \\
\hline$|\mathrm{B}|$ & $0.0622 \mathrm{~T}$ & $0.0900 \mathrm{~T}$ & Motor:-y & over \\
\hline$\Delta \mathrm{Bx} / \Delta \mathrm{x}$ & $0.0564 \mathrm{~T} / \mathrm{m}$ & $0.0187 \mathrm{~T} / \mathrm{m}$ & \multirow{3}{*}{$\begin{array}{c}\text { Motor:-y } \\
\text { Motor: Center } \\
\text { Motor: Center }\end{array}$} & \multirow{3}{*}{$\begin{array}{l}\text { under } \\
\text { over } \\
\text { over }\end{array}$} \\
\hline$\Delta \mathrm{Bx} / \Delta \mathrm{y}$ & $-0.0038 \mathrm{~T} / \mathrm{m}$ & $-0.0085 \mathrm{~T} / \mathrm{m}$ & & \\
\hline$\Delta \mathrm{Bx} / \Delta \mathrm{z}$ & $-0.1168 \mathrm{~T} / \mathrm{m}$ & $-0.3543 \mathrm{~T} / \mathrm{m}$ & & \\
\hline$\Delta \mathrm{By} / \Delta \mathrm{x}$ & $0.0783 \mathrm{~T} / \mathrm{m}$ & $0.0010 \mathrm{~T} / \mathrm{m}$ & \multirow{3}{*}{$\begin{array}{c}\text { Motor:-y } \\
\text { Motor: Center } \\
\text { Motor: Center }\end{array}$} & \multirow{3}{*}{$\begin{array}{l}\text { under } \\
\text { under } \\
\text { under }\end{array}$} \\
\hline$\Delta \mathrm{By} / \Delta \mathrm{y}$ & $0.0061 \mathrm{~T} / \mathrm{m}$ & $-0.0033 \mathrm{~T} / \mathrm{m}$ & & \\
\hline$\Delta \mathrm{By} / \Delta \mathrm{z}$ & $0.0737 \mathrm{~T} / \mathrm{m}$ & $-0.0630 \mathrm{~T} / \mathrm{m}$ & & \\
\hline$\Delta \mathrm{Bz} / \Delta \mathrm{x}$ & $0.0918 \mathrm{~T} / \mathrm{m}$ & $-0.4872 \mathrm{~T} / \mathrm{m}$ & \multirow{3}{*}{\begin{tabular}{c|} 
Motor:-y \\
Motor: Center \\
Motor: Center
\end{tabular}} & \multirow{3}{*}{$\begin{array}{l}\text { over } \\
\text { over } \\
\text { under }\end{array}$} \\
\hline$\Delta \mathrm{Bz} / \Delta \mathrm{y}$ & $0.0047 \mathrm{~T} / \mathrm{m}$ & $0.0131 \mathrm{~T} / \mathrm{m}$ & & \\
\hline$\Delta \mathrm{Bz} / \Delta \mathrm{z}$ & $0.0896 \mathrm{~T} / \mathrm{m}$ & $-0.0148 \mathrm{~T} / \mathrm{m}$ & & \\
\hline & $-0.1356 \mathrm{~T} / \mathrm{m}$ & $-0.4872 \mathrm{~T} / \mathrm{m}$ & \multirow{3}{*}{\begin{tabular}{|c|} 
Motor:-y \\
Motor: \\
Center \\
Motor: Center \\
\end{tabular}} & \multirow{3}{*}{$\begin{array}{l}\text { over } \\
\text { over } \\
\text { under }\end{array}$} \\
\hline$\Delta B y z / \Delta y$ & $-0.0037 \mathrm{~T} / \mathrm{m}$ & $0.0131 \mathrm{~T} / \mathrm{m}$ & & \\
\hline$\Delta \mathrm{Byz} / \Delta \mathrm{z}$ & $-0.1160 \mathrm{~T} / \mathrm{m}$ & $-0.0162 \mathrm{~T} / \mathrm{m}$ & & \\
\hline $\begin{array}{l}\text { Operated in fiel } \\
\text { Byz is the field } \\
\text { single most inflt } \\
\text { Bx is the field th } \\
\text { Field properties } \\
\text { behavior }\end{array}$ & $\begin{array}{l}\text { up to } 2 x \text { predicted } \\
\text { at is perpendicular to } \\
\text { ntial field property to } \\
\text { is parallel to the cry } \\
\text { Bold are the most }\end{array}$ & $\begin{array}{l}\text { pendicular AMS }-02 \text { fields } \\
\text { le cryocooler axis, and is the } \\
\text { e cryocooler's behavior. } \\
\text { ooler axis } \\
\text { uential to the cryocooler's }\end{array}$ & & \\
\hline
\end{tabular}

Table 1: Chart comparing predicted AMS-02 magnetic field to the magnetic field that the M87N was exposed to. 


\section{Figures}

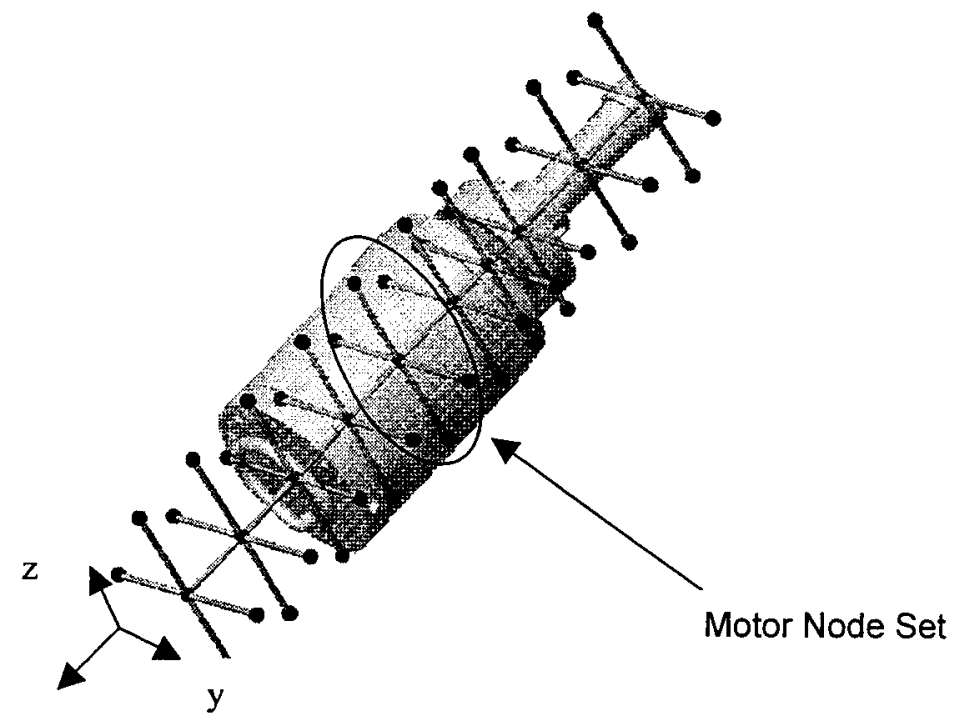

$\mathrm{x}$

Figure 1 


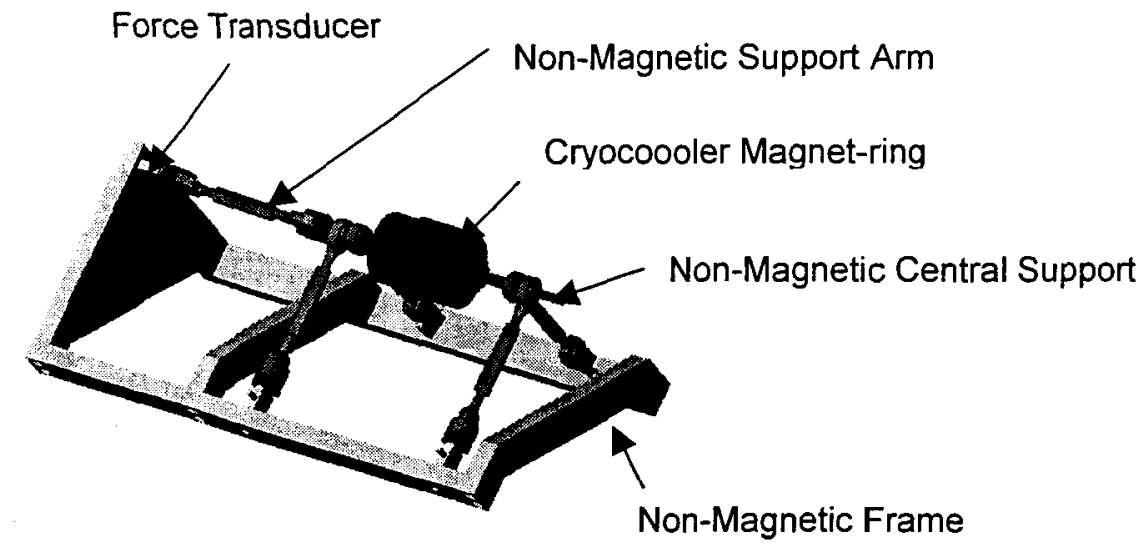

Figure 2 


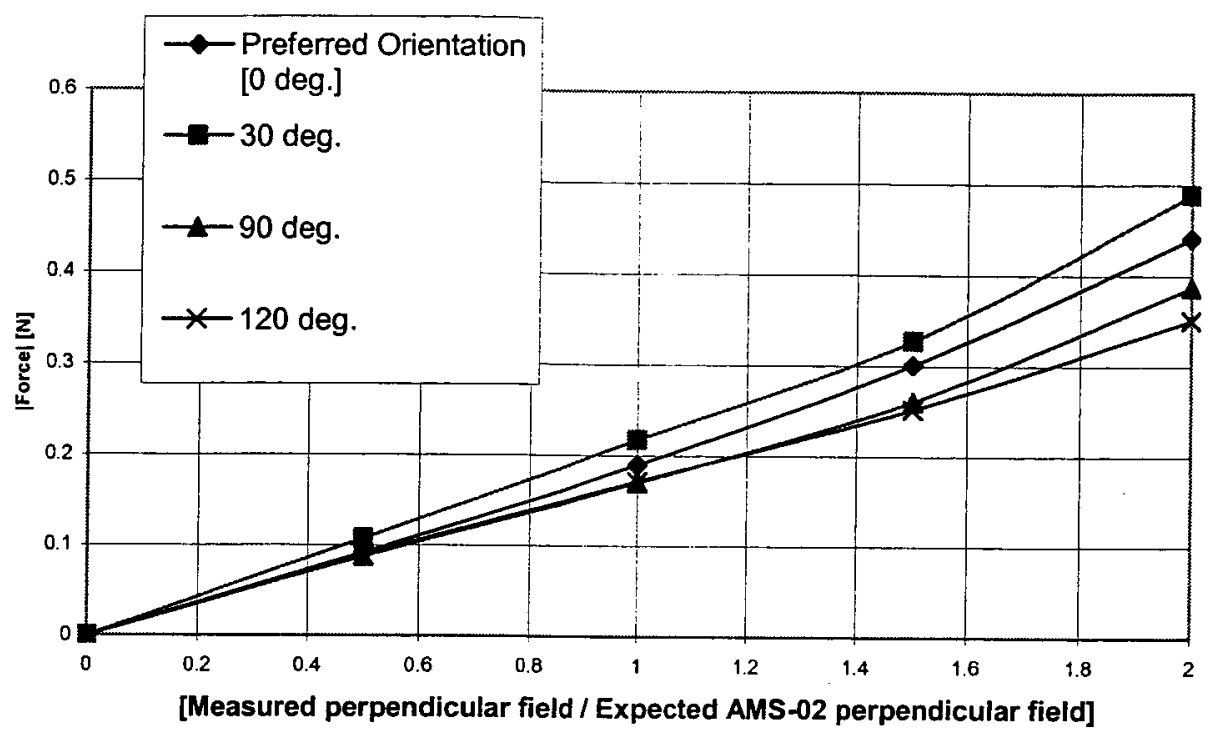

Figure 3 


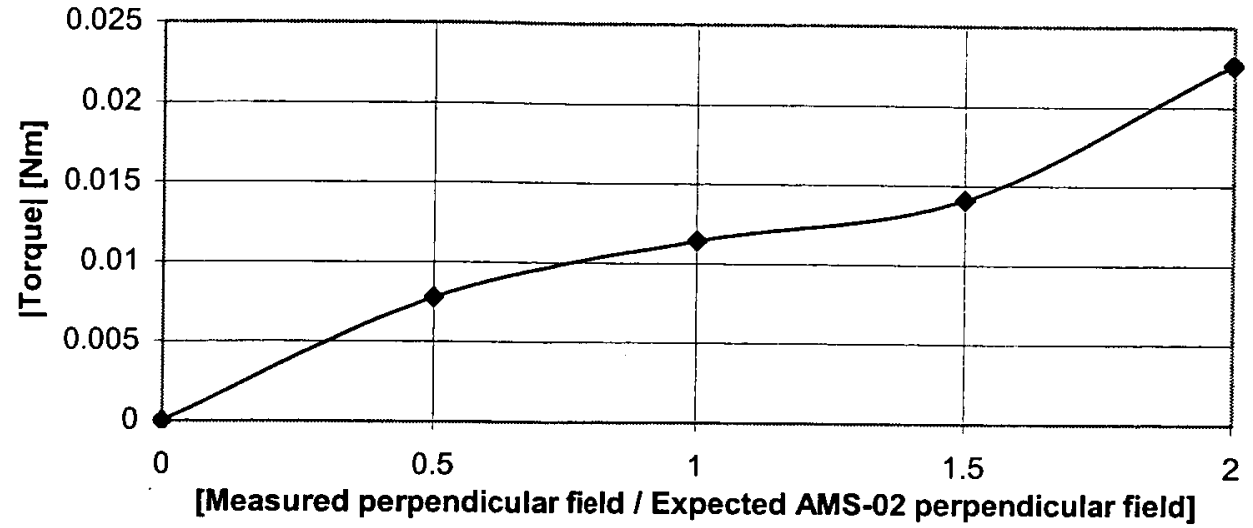

Figure 4 


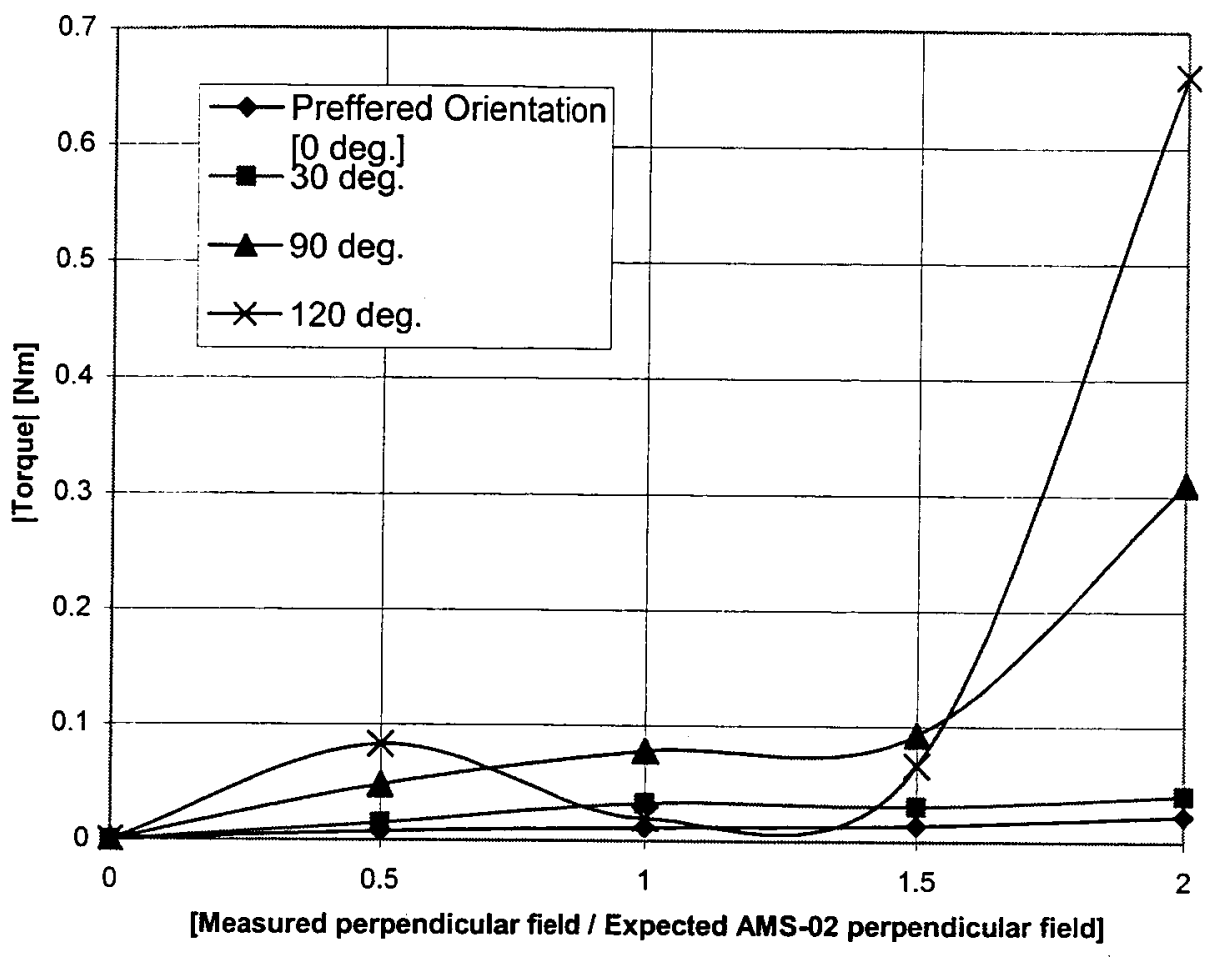

Figure 5 


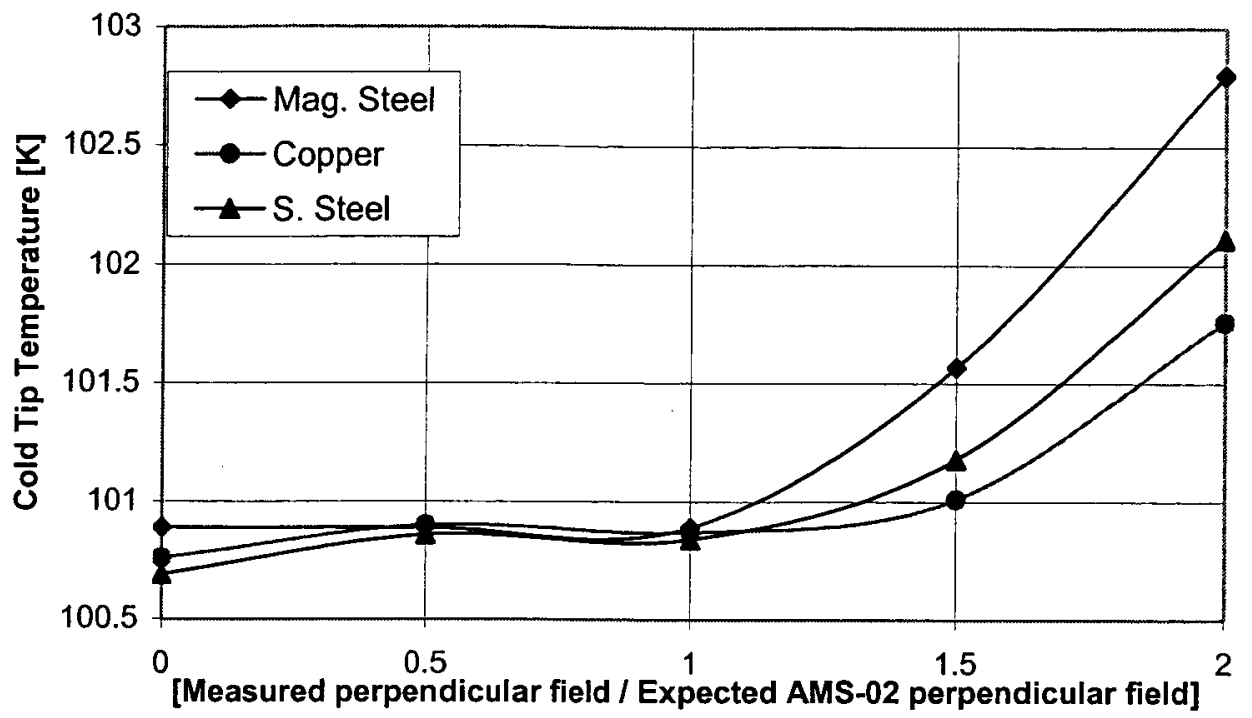

Figure 6 


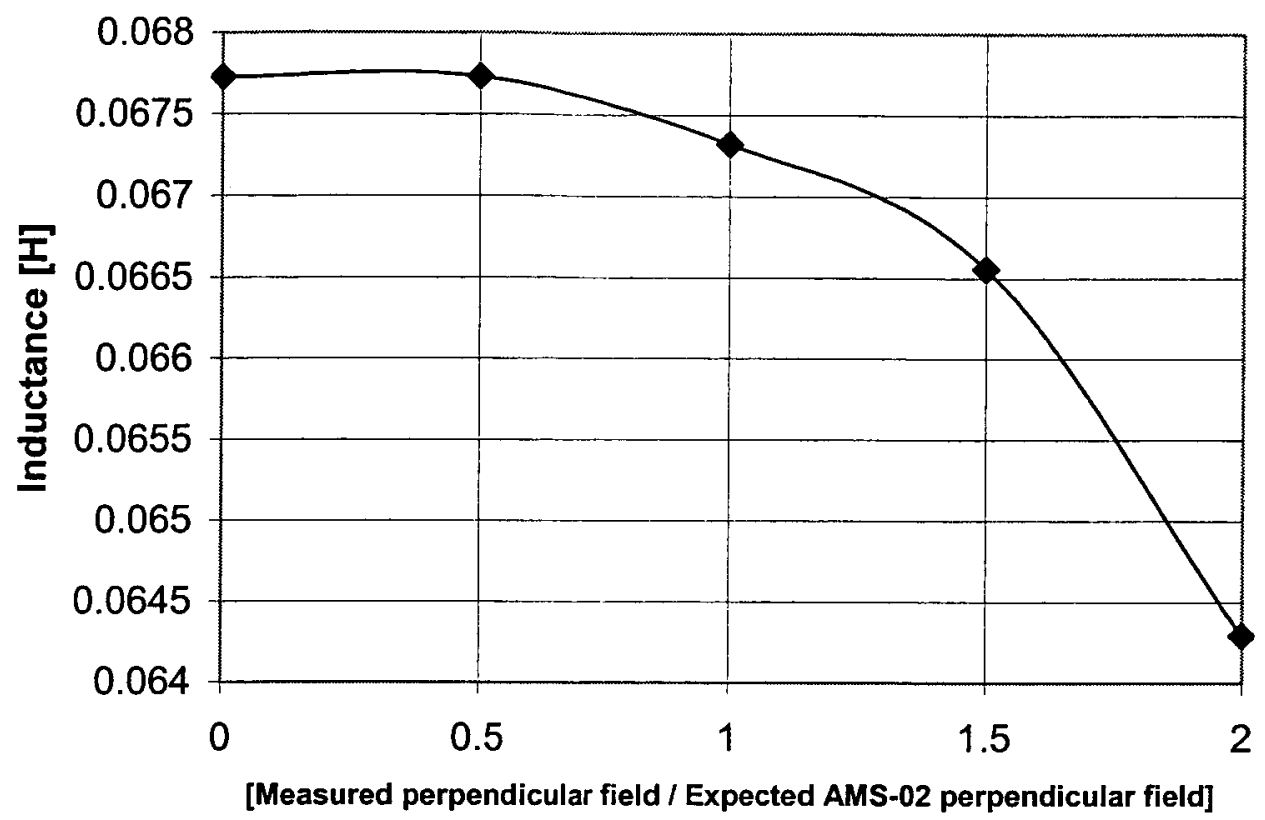

Figure 7 


\section{Captions for Figures}

Figure 1: Cryocooler based co-ordinate system and node matrix

Figure 2: Test rig to measure forces and torques imparted by an external magnetic field on the M87N magnet-ring.

Figure 3: Net magnetic force on the M87N magnet-ring assembly as a function of the applied magnetic field for various orientations of the magnet-ring with respect to the preferred orientation.

Figure 4: Net magnetic torque on magnet-ring assembly at preferred orientation as a function of applied field.

Figure 5: Net magnetic torque on magnet-ring assembly at preferred orientation as a function of applied field for various orientations of the magnet-ring.

Figure 6: Cold tip temperatures as a function of the applied magnetic field for balancers made of different materials.

Figure 7: The inductance of the $\mathrm{M} 87 \mathrm{~N}$ motor as a function of the applied magnetic field. 